\title{
Evaluation of neuroactive steroid levels by liquid chromatography-tandem mass spectrometry in central and peripheral nervous system: Effect of diabetes
}

\author{
Donatella Caruso ${ }^{a}$, Samuele Scurati ${ }^{a}$, Omar Maschi $^{\text {a }}$, Leonardo De Angelis ${ }^{\text {a }}$, \\ Ilaria Roglio ${ }^{\mathrm{b}}$, Silvia Giatti ${ }^{\mathrm{b}}$, Luis Miguel Garcia-Segura ${ }^{\mathrm{c}}$, Roberto C. Melcangi ${ }^{\mathrm{b}, *}$ \\ ${ }^{a}$ Department of Pharmacological Sciences, University of Milan, Milano, Italy \\ ${ }^{\mathrm{b}}$ Department of Endocrinology and Center of Excellence on Neurodegenerative Diseases, University of Milan, Via Balzaretti 9 , 20133 Milano, Italy \\ ${ }^{\mathrm{c}}$ Instituto Cajal, C.S.I.C., Madrid, Spain
}

Received 28 February 2007; received in revised form 11 June 2007; accepted 11 June 2007

\begin{abstract}
The nervous system is a target for physiological and protective effects of neuroactive steroids. Consequently, the assessment of their levels in nervous structures under physiological and pathological conditions is a top priority. To this aim, identification and quantification of pregnenolone (PREG), progesterone (PROG), dihydroprogesterone (DHP), tetrahydroprogesterone (THP), testosterone (T), dihydrotestosterone (DHT), 5 $\alpha$ androstan-3 $\alpha, 17 \beta$-diol ( $3 \alpha$-diol), 17 $\alpha$ - and 17 $\beta$-estradiol (17 $\alpha$-E and $17 \beta$-E) by liquid chromatography and tandem mass spectrometry (LC-MS/ MS) has been set up. After validation, this method was applied to determine the levels of neuroactive steroids in central (i.e., cerebral cortex, cerebellum and spinal cord) and peripheral (i.e., brachial nerve) nervous system of control and diabetic rats. In controls only the brachial nerve had detectable levels of all these neuroactive steroids. In contrast, $17 \alpha-\mathrm{E}$ in cerebellum, $17 \alpha-\mathrm{E}, 17 \beta-\mathrm{E}, \mathrm{DHP}$ and THP in cerebral cortex, and $17 \alpha-\mathrm{E}$, $17 \beta-\mathrm{E}$ and DHP in spinal cord were under the detection limit. Diabetes, induced by injection with streptozotocin, strongly affected the levels of some neuroactive steroids. In particular, the levels of PREG, PROG and T in cerebellum, of PROG, T and $3 \alpha$-diol in cerebral cortex, of PROG, DHT and $3 \alpha$-diol in spinal cord and of PREG, DHP, THP, T, DHT and $3 \alpha$-diol in brachial nerve were significantly decreased. In conclusion, the data here reported demonstrate that the LC-MS/MS method allows the assessment of neuroactive steroids in the nervous system with high sensitivity and specificity and that diabetes strongly affects their levels, providing a further basis for new therapeutic tools based on neuroactive steroids aimed at counteracting diabetic neuropathy.
\end{abstract}

(C) 2007 Published by Elsevier Ltd.

Keywords: Streptozotocin; Central nervous system; Peripheral nervous system; Steroid level; Rat; Neuroprotection

\section{Introduction}

One important complication of diabetes is the damage that may occur at the level of the nervous system. Diabetic peripheral neuropathy occurs in $60-70 \%$ of patients affected by type I and type II diabetes and, as described both in human and in animal models. For instance streptozotocin (STZ)-induced neuropathy, is associated with a spectrum of functional (e.g., nerve conduction velocity, expression of myelin proteins, $\mathrm{Na}^{+}, \mathrm{K}^{+}$-ATPase activity, nociceptive threshold, etc.) and

\footnotetext{
* Corresponding author. Tel.: +3902 50318238; fax: +390250318204.

E-mail address: roberto.melcangi@unimi.it (R.C. Melcangi).
}

structural (e.g., axonal degeneration, paranodal demyelination and loss of myelinated fibers) changes in peripheral nerves (Yagihashi, 1997; Biessels et al., 1999; Bianchi et al., 2004; Veiga et al., 2006; Leonelli et al., 2007). Moreover, the impact of diabetes on the central nervous system (CNS) is well recognized. Neurophysiological and structural changes at the level of cerebral areas, such as hypothalamus, cerebral cortex, cerebellum and hippocampus, are associated with cognitive deficits and increased risk of dementia, stroke, cerebrovascular and Alzheimer disease and psychiatric disorders, such as depression and eating disorders (Gispen and Biessels, 2000; Jacobson et al., 2002; Sima et al., 2004; van Harten et al., 2006).

It is well known that neuroactive steroids, like for instance pregnenolone (PREG), progesterone (PROG), and its deriva- 
tives dihydroprogesterone (DHP) and tetrahydroprogesterone (THP), testosterone (T) and its derivatives dihydrotestosterone (DHT) and $5 \alpha$-androstan- $3 \alpha, 17 \beta$-diol ( $3 \alpha$-diol), and estrogens regulate several physiological processes in neurons and glial cells of the peripheral nervous system (PNS) and CNS (Melcangi et al., 2001, 2002, 2005; Garcia-Segura and Melcangi, 2006). Moreover, recent observations obtained in our and other laboratories have indicated that these neuroactive steroids exert protective effects in several experimental models of neurodegeneration (Lapchak and Araujo, 2001; Azcoitia et al., 2003; McCullogh and Hurn, 2003; Ciriza et al., 2004; Griffin et al., 2004), including diabetic neuropathy (Veiga et al., 2006; Leonelli et al., 2007; Saravia et al., 2006). Interestingly, the impact of diabetes is also evident on steroid levels. Indeed, several observations have shown dysfunction in the reproductive axis associated with diabetes, with modifications of sex steroid plasma levels (El'tseva et al., 1993; Sudha et al., 2000; Tanaka et al., 2001; van Dam et al., 2003; Salonia et al., 2006). Moreover, we have recently observed that STZ-induced diabetes causes plasma PROG levels to drop steeply in male rats (Leonelli et al., 2007). Furthermore, altered levels of neuroactive steroids occur not only in plasma but also in nervous tissues. It has been recently reported that an increase of PROG biosynthesis concomitant with a decrease of formation of its metabolite, THP, occurs in the spinal cord of rats with STZ-induced diabetes (Saredi et al., 2005). This single report is highly relevant because the modifications of the levels of steroids in neural tissue with diabetes may potentially increase, decrease and/or be the consequence of local pathological damage and may affect the result of therapies based on neuroactive steroids. Consequently, it is extremely important to determine the levels of neuroactive steroids in central and peripheral nervous structures and to assess whether these levels are modified by diabetes.

With this aim, an analytical method based on liquid chromatography and tandem mass spectrometry (LC-MS/MS) for the identification and quantification of PREG, PROG, DHP, THP, T, DHT, $3 \alpha$-diol, $17 \alpha$ - and $17 \beta$-estradiol $(17 \alpha-\mathrm{E}$ and $17 \beta$ E) has been set up. After validation of the LC-MS/MS procedure, this method was applied to the identification and quantitative determination of the neuroactive steroids mentioned above in CNS structures, such as cerebral cortex, cerebellum and spinal cord, and in a peripheral nerve, such as brachial nerve, of control and STZ-treated rats.

\section{Experimental procedures}

\subsection{Materials}

5-Pregnen-3 $\beta$-ol-20-one (PREG), progesterone (PROG), 5 $\alpha$-pregnane-3, 20-dione (DHP), $3 \alpha$-hydroxy-5 $\alpha$-pregnen-20-one (THP), testosterone (T), $5 \alpha$ -

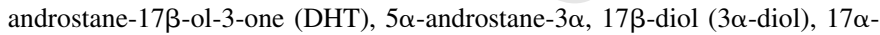
and $17 \beta$-estradiol $(17 \alpha-\mathrm{E}$ and $17 \beta$-E), were purchased from Sigma-Aldrich. 17,21,21,21-D 4 PREG (D4-PREG) was kindly synthesized by Dr. P. Ferraboschi (Deptartment of Medical Chemistry, Biochemistry and Biotechnology, University of Milano, Italy); 2,2,4,6,6-17 $\alpha, 21,21,21$ - $_{9}$-PROG ( D $_{9}$-PROG) was obtained from Medical Isotopes (Pelham, NH, USA) and 2,4,16,16- $\mathrm{D}_{4}-$ $17 \beta-E\left(D_{4}-17 \beta-E\right)$ from CDN Isotope Pointe-Claire (Que., Canada). SPE cartridges (Discovery DS-C18 $500 \mathrm{mg}$ ) were from Supelco, Italy. All solvents and reagents were HPLC grade (Sigma-Aldrich, Italy).

\section{2. $L C-M S / M S$ analysis}

Positive atmospheric pressure chemical ionization (APCI+) experiments were performed using a linear ion trap-mass spectrometer (LTQ, ThermoElectron Co., San Jose, CA, USA) equipped with a Surveyor liquid chromatography (LC) Pump Plus and a Surveyor Autosampler Plus (ThermoElectron Co., San Jose, CA, USA).

The LC mobile phases were (A) $\mathrm{H}_{2} \mathrm{O} / 0.1 \%$ formic acid and (B) methanol $(\mathrm{MeOH}) / 0.1 \%$ formic acid. The gradient (flow rate $1 \mathrm{ml} / \mathrm{min}$ ) was as follows: $T_{0}$ $60 \% \mathrm{~A}, T_{4.5} 60 \% \mathrm{~A}, T_{5} 40 \% \mathrm{~A}, T_{8} 40 \% \mathrm{~A}, T_{45} 30 \% \mathrm{~A}, T_{46} 10 \% \mathrm{~A}, T_{54} 10 \% \mathrm{~A}, T_{55}$ $60 \% \mathrm{~A}, T_{65} 60 \% \mathrm{~A}$. The split valve was set at $0-10 \mathrm{~min}$ to waste, $10-50 \mathrm{~min}$ to source and 50-65 min to waste. The Inertsil ODS-2 RP-C 18 column $(5 \mu \mathrm{m}$, $150 \mathrm{~mm} \times 4.6 \mathrm{~mm}$ i.d.; GL Sciences Inc., Japan) was maintained at $40{ }^{\circ} \mathrm{C}$. The injection volume was $25 \mu \mathrm{l}$ and the injector needle was washed with $\mathrm{MeOH} /$ water $1 / 1(\mathrm{v} / \mathrm{v})$. Peaks of the LC-MS/MS were evaluated using a Dell workstation by means of the software Excalibur ${ }^{\circledR}$ release 2.0 SR2 (ThermoElectron Co, San Jose, CA, USA)

The mass spectrometer was operated in the positive ion mode with the atmospheric pressure chemical ionization (APCI) source using nitrogen as sheath, auxiliary and sweep gas at flow rates of 23, 8, 2 (arbitrary units), respectively. Other ion-source parameters: vaporizer temperature $450{ }^{\circ} \mathrm{C}$, ion-source collisionenergy (SID) $20 \mathrm{~V}$, capillary temperature $275^{\circ} \mathrm{C}$. The mass spectrometer was employed in MS/MS mode using helium as collision gas. The relative collisionenergy was set at $35 \%$ for $17 \alpha-\mathrm{E}, 17 \beta-\mathrm{E}, \mathrm{D}_{4}-17 \beta-\mathrm{E}, 3 \alpha$-diol and at $35 \%$ using the Wide Band Activation mode (ThermoElectron Co., USA) for all the other steroids. Samples were analyzed employing the transitions reported in Table 1.

\subsection{Study design and sample preparation}

Two-month-old male Sprague-Dawley rats, Crl:CD BR (Charles River, Italy) were housed in the animal quarters of the Department of Endocrinology at the University of Milan with controlled temperature and humidity. The light schedule was $14 \mathrm{~h}$ light and $10 \mathrm{~h}$ dark (lights on at $6: 30 \mathrm{~h}$ ). The animals were handled following the European Union Normative (Council Directive 86/609/ EEC guidelines), with the approval of our Institutional Animal Use and Care Committees. Rats were randomly divided into two groups (control and diabetes).

Diabetes was induced by a single injection into the tail vein of freshly prepared STZ (65 mg/kg; Sigma, Italy) in citrate buffer $0.09 \mathrm{M} \mathrm{pH} 4.8$. Control animals were injected with $0.09 \mathrm{M}$ citrate buffer at $\mathrm{pH} 4.8$. Hyperglycemia was confirmed $48 \mathrm{~h}$ after STZ injection by measuring tail vein blood glucose levels using a Glucomen tester (Menarini, Italy). Only animals with mean plasma glucose levels above $300 \mathrm{mg} / \mathrm{dl}$ were classified as diabetic. Three months after the diabetes induction, rats were sacrificed and cerebral cortex, cerebellum, spinal cord and brachial nerve were collected, weighed and stored at $-80{ }^{\circ} \mathrm{C}$ before the analysis.

Table 1

Analytical parameters

\begin{tabular}{llllll}
\hline & $\begin{array}{l}\text { Precursor } \\
\text { ions }\end{array}$ & $\begin{array}{l}\text { Transition } \\
\text { monitore }\end{array}$ & RRT & IS & Segment \\
\hline $\mathrm{D}_{4}-17 \beta-\mathrm{E}$ & 259 & 161 & 1 & - & 1 \\
$17 \beta-\mathrm{E}$ & 255 & 133,159 & 1.01 & $\mathrm{D}_{4}-17 \beta-\mathrm{E}$ & 1 \\
$17 \alpha-\mathrm{E}$ & 255 & 133,159 & 1.08 & $\mathrm{D}_{4}-17 \beta-\mathrm{E}$ & 1 \\
$\mathrm{~T}$ & 289 & 97,109 & 1.07 & $\mathrm{D}_{4}-17 \beta-\mathrm{E}$ & 1 \\
$\mathrm{D}_{9}$-PROG & 324 & 100 & 1 & - & 2 \\
$\mathrm{PROG}$ & 315 & 97,109 & 1.02 & $\mathrm{D}_{9}$-PROG & 2 \\
$\mathrm{DHT}$ & 291 & 255 & 0.89 & $\mathrm{D}_{9}$-PROG & 2 \\
$3 \alpha-$ Diol & 257 & $121,135,147$, & 1.08 & $\mathrm{D}_{9}$-PROG & 2 \\
& & 161,175 & & & \\
$\mathrm{D}_{4}$-PREG & 303 & 175 & 1 & - & 3 \\
PREG & 299 & 159,199 & 1.01 & $\mathrm{D}_{4}$-PREG & 3 \\
DHP & 299 & 189 & 1.05 & $\mathrm{D}_{4}$-PREG & 3 \\
THP & 301 & $159,173,187$ & 1.27 & $\mathrm{D}_{4}$-PREG & 3 \\
\end{tabular}

RRT: relative retention time (calculated against the IS monitored in the corresponding segment); IS: internal standard. 
The samples were extracted and purified according to Vallée and collaborators (Vallée et al., 2000) with minor modification. Briefly, samples $(100 \mathrm{mg} /$ tissue $)$ were added with internal standards and homogenized in $2 \mathrm{ml}$ of $\mathrm{MeOH} /$ acetic acid (99:1, v/v) using an ultrasonic homogenizer (Bransonic Ultrasonic Co., USA). After an overnight at $4{ }^{\circ} \mathrm{C}$, samples were centrifuged at 12,000 rpm for $5 \mathrm{~min}$ and the pellet was extracted twice with $1 \mathrm{ml}$ of $\mathrm{MeOH} /$ acetic acid (99:1, v/v). The organic phases were combined and dried with a gentle stream of nitrogen in a $40{ }^{\circ} \mathrm{C}$ water bath. The samples were resuspended with $3 \mathrm{ml}$ of $\mathrm{MeOH} / \mathrm{H}_{2} \mathrm{O}(10: 90$, v/v) and passed through SPE cartridge, previously activated with $\mathrm{MeOH}(5 \mathrm{ml})$ and $\mathrm{MeOH}: \mathrm{H}_{2} \mathrm{O}$ 10:90 (v/v) $(5 \mathrm{ml})$. The steroids were eluted in $\mathrm{MeOH}$, concentrated and transferred in autosampler vials before the LC-MS/MS analysis.

\subsection{Quantitative analysis and analytical method validation}

\subsubsection{Calibration curves}

Quantitative analysis was performed on the basis of calibration curves daily prepared and analyzed: blank samples (6\% albumin in PBS) were spiked with $\mathrm{D}_{4}-17 \beta$-E (1 ng/sample), $\mathrm{D}_{9}$-PROG (0.2 ng/sample) and $\mathrm{D}_{4}$-PREG (5 ng/sample), as internal standards. Increasing amounts $(0.05-5 \mathrm{ng} / \mathrm{sample})$ of each steroid were added. Calibration curves were extracted and analyzed as already described for samples.

\subsubsection{Limit of quantification, precision and accuracy}

The limit of quantification (LOQ) was calculated as the lowest amount of steroid measured with a minimum error of $\pm 20 \%$ in triplicate, as described by Vallée and collaborators (Vallée et al., 2000).

Inter-assay accuracy and reproducibility of the method were calculated over a series of blank samples spiked with 0.5, 2.5 and $5 \mathrm{ng} / \mathrm{sample}$ and estimated on the basis of calibration curves. Accuracy was calculated by the ratio (obtained value/true value $\times 100$ ) in different five samples prepared and injected in duplicate in different days. Precision was determined as coefficient of variation $(\mathrm{CV} \%)$ calculated on the basis of five samples prepared and injected in different days.

\subsection{Statistical analysis}

The linearity of the standard curve $\left(r^{2}\right)$, the accuracy $(\%)$ and the precision (CV\%) inter-series were judged by GraphPad4 PRISM (version 4). Student's $t$ test was used to determine significant differences between control and diabetic tissues.

\section{Results}

The present approach is based on the power of tandem mass spectrometry. The increase of specificity (also reflecting an improvement of sensitivity) achieved with this method represents the basis for unambiguous identification of the analytes. Their structural identification is based not only on the molecular ion and retention time, but also on specific fragmentation routes specifically related to the structure. Therefore, the described method allows the identification and quantification of PREG, PROG, DHP, THP, T, DHT, $3 \alpha$-diol, $17 \alpha$-E and $17 \beta$-E in nervous tissues with satisfactory standards of linearity, precision, accuracy and sensitivity (Table 2). The correlation coefficient values $\left(r^{2}\right)$ were greater than 0.99, indicating an adequate linearity of our analytical procedure. As shown in this table, accuracy and reproducibility were within the accepted tolerances even at the lowest concentration level studied $(93-108 \%$; CV\% < 15). As expected, the highest values were obtained at the lower concentrations and for DHP, for which the LOQ is very high due to a difficulty in the ionization.

Fig. 1 shows representative examples of ion chromatograms and the mean of five calibration curves prepared and analyzed in different days. In the first segment of the analysis (1418 min, panel A) $17 \alpha-\mathrm{E}, 17 \beta-\mathrm{E}$ and $\mathrm{T}$ were detected. These compounds were quantified using $\mathrm{D}_{4}-17 \beta-\mathrm{E}$ as internal standard; the linearity of the determinations was presented in the right part of the panel A. Similarly, panels B and C show the second (18-32 $\mathrm{min}$ ) and the third (32-47 min) segments of the analysis and the respective calibration curves. All the compounds were discernible on the basis of different ion and/or retention times.

Fig. 2 shows the levels of neuroactive steroids in cerebellum of control and STZ-treated rats. All neuroactive steroids analyzed, with the exception of $17 \alpha-\mathrm{E}$, were identified and measured in control animals. A significant impact of three months of diabetes was evident. In particular, PREG, PROG and T levels were significantly decreased in cerebellum of STZtreated rats. On the contrary, metabolites of PROG (i.e., DHP and THP) or of T (i.e., DHT and $3 \alpha$-diol) as well as the levels of $17 \beta-E$ were unaffected by diabetes. A different pattern of steroid levels in control and STZ-rats was present in the other two CNS regions analyzed. In particular, as shown in Fig. 3, only PREG, PROG, T and its derivatives (i.e., DHT and $3 \alpha$-diol) were detected in the cerebral cortex of control animals. Among

Table 2

Validation of the method

\begin{tabular}{|c|c|c|c|c|c|c|c|c|}
\hline & \multirow[t]{2}{*}{$r^{2}$} & \multirow[t]{2}{*}{ LOQ (pg/sample) } & \multicolumn{2}{|l|}{ Level 0.5} & \multicolumn{2}{|l|}{ Level 2.5} & \multicolumn{2}{|l|}{ Level 5} \\
\hline & & & Accuracy (\%) & Precision CV\% & Accuracy (\%) & Precision CV\% & Accuracy $(\%)$ & Precision CV\% \\
\hline PREG & 0.999 & 0.05 & 96 & 11.91 & 100 & 6.71 & 101 & 1.79 \\
\hline PROG & 0.990 & 0.05 & 105 & 12.23 & 98 & 6.97 & 101 & 1.85 \\
\hline DHP & 0.990 & 0.25 & 101 & 22.06 & 102 & 7.80 & 100 & 2.58 \\
\hline THP & 0.998 & 0.1 & 93 & 0.56 & 96 & 0.16 & 101 & 0.44 \\
\hline $\mathrm{T}$ & 0.999 & 0.02 & 109 & 8.06 & 102 & 5.45 & 100 & 0.64 \\
\hline DHT & 0.992 & 0.05 & 102 & 9.61 & 100 & 3.90 & 100 & 1.67 \\
\hline $3 \alpha$-Diol & 0.992 & 0.05 & 107 & 11.07 & 94 & 4.58 & 101 & 1.83 \\
\hline $17 \alpha-E$ & 0.999 & 0.02 & 105 & 4.74 & 102 & 2.79 & 100 & 0.65 \\
\hline $17 \beta-E$ & 0.999 & 0.02 & 102 & 3.12 & 102 & 2.78 & 99 & 0.67 \\
\hline
\end{tabular}

$r^{2}$ : Linearity of the assay calculated on the basis of almost four calibration curves; LOQ: limit of quantification; accuracy is calculated as obtained value/true value $\times 100$ (five samples prepared and injected in different days) on the basis of the calibration curves; precision is calculated as CV\% (five samples prepared and injected in different days). 

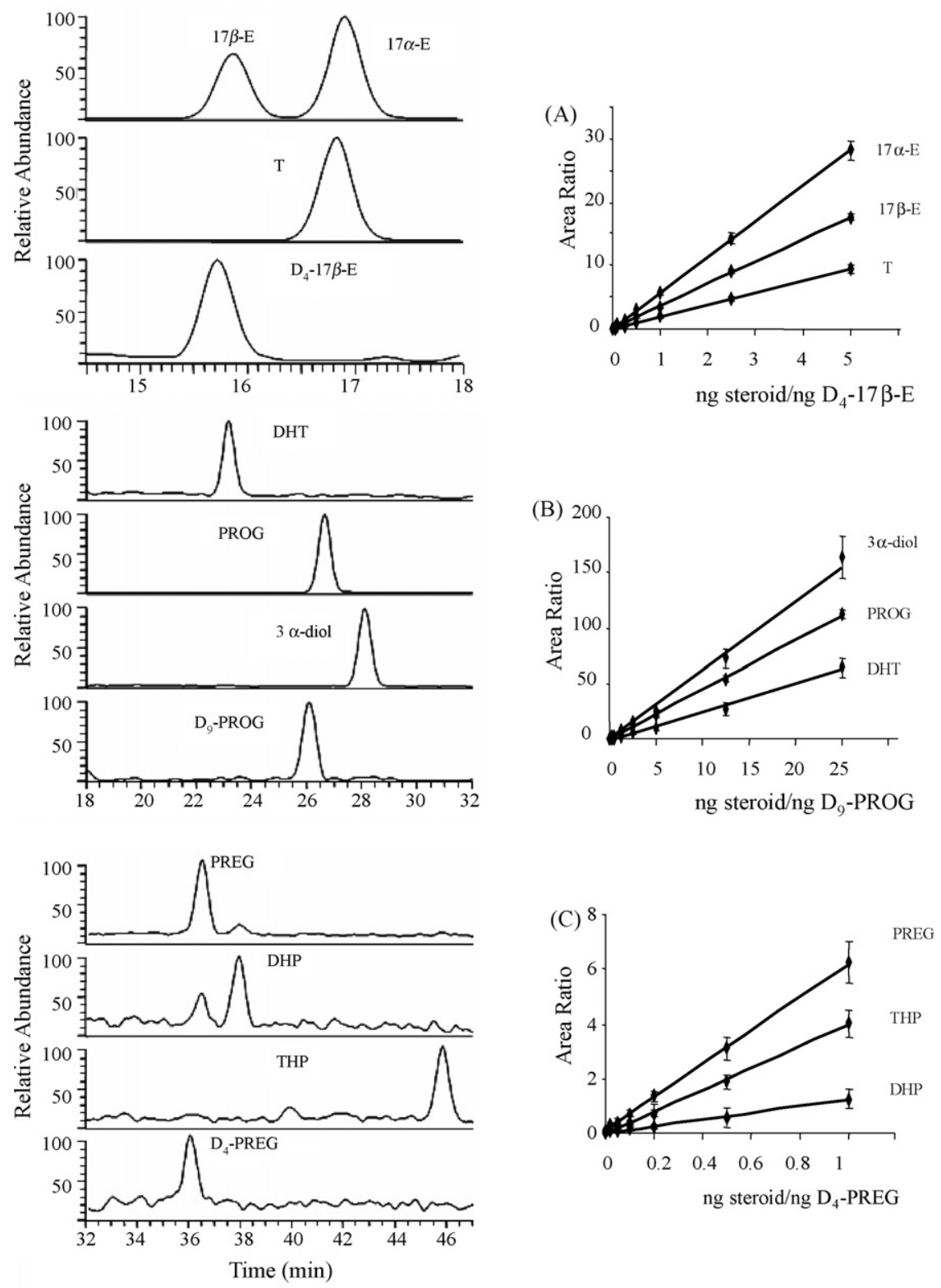

Fig. 1. Representative LC-MS/MS chromatogram of a standard sample. The identity of each peak is based on the retention time and MS/MS spectra of authentic compounds. Calibration curves were prepared as described in Section 2. Data are expressed as mean \pm S.E.M. $(n=5)$. (Panel A) Left: first segment of the analysis (13-18 min) $17 \alpha-\mathrm{E}$ and $17 \beta-\mathrm{E}$, T and $\mathrm{D}_{4}-17 \beta-\mathrm{E}$ as internal standard; right: corresponding calibration curves. Panel (B) left: second segment of the analysis (18$32 \mathrm{~min}$ ) DHT, PROG, $3 \alpha$-diol and $\mathrm{D}_{9}$-PROG as internal standard; right: corresponding calibration curves. (Panel C) Left: third segment of the analysis (32-47 min) PREG, THP, DHP and $\mathrm{D}_{4}$-PREG as internal standard; right: corresponding calibration curves.

these, only the levels of PROG, T and $3 \alpha$-diol were significantly decreased by diabetes. The levels of DHT observed in the cerebral cortex of STZ-treated rats were under the detection limit, but not significantly different from those observed in control rats. It is interesting to note that in the cerebral cortex of both control and diabetic rats, the levels of DHP, THP, $17 \alpha-\mathrm{E}$ and $17 \beta-\mathrm{E}$ were under the detection limit.

Most steroids analyzed were detected and measured in the spinal cord of control and diabetic animals, with the exception of DHP, $17 \alpha-\mathrm{E}$ and $17 \beta-\mathrm{E}$ (Fig. 4). PROG, DHT and $3 \alpha$-diol were significantly decreased in the spinal cord of diabetic animals, while PREG, T, and THP were unaffected by diabetes. Interestingly, the levels of $17 \beta-\mathrm{E}$, which were under the detection limit in the spinal cord of control animals, were detectable in diabetic animals.

As shown in Fig. 5, brachial nerves of control animals showed detectable levels of all neuroactive steroids and the impact of diabetes was evident with most of them, with the exception of PROG, $17 \alpha-E$ and $17 \beta-E$. In particular, PREG, DHP, THP, T, DHT and $3 \alpha$-diol levels decreased significantly in brachial nerve of diabetic animals.

\section{Discussion}

The advent of robust and analytically reliable techniques based on the combination of liquid chromatography (LC) and 

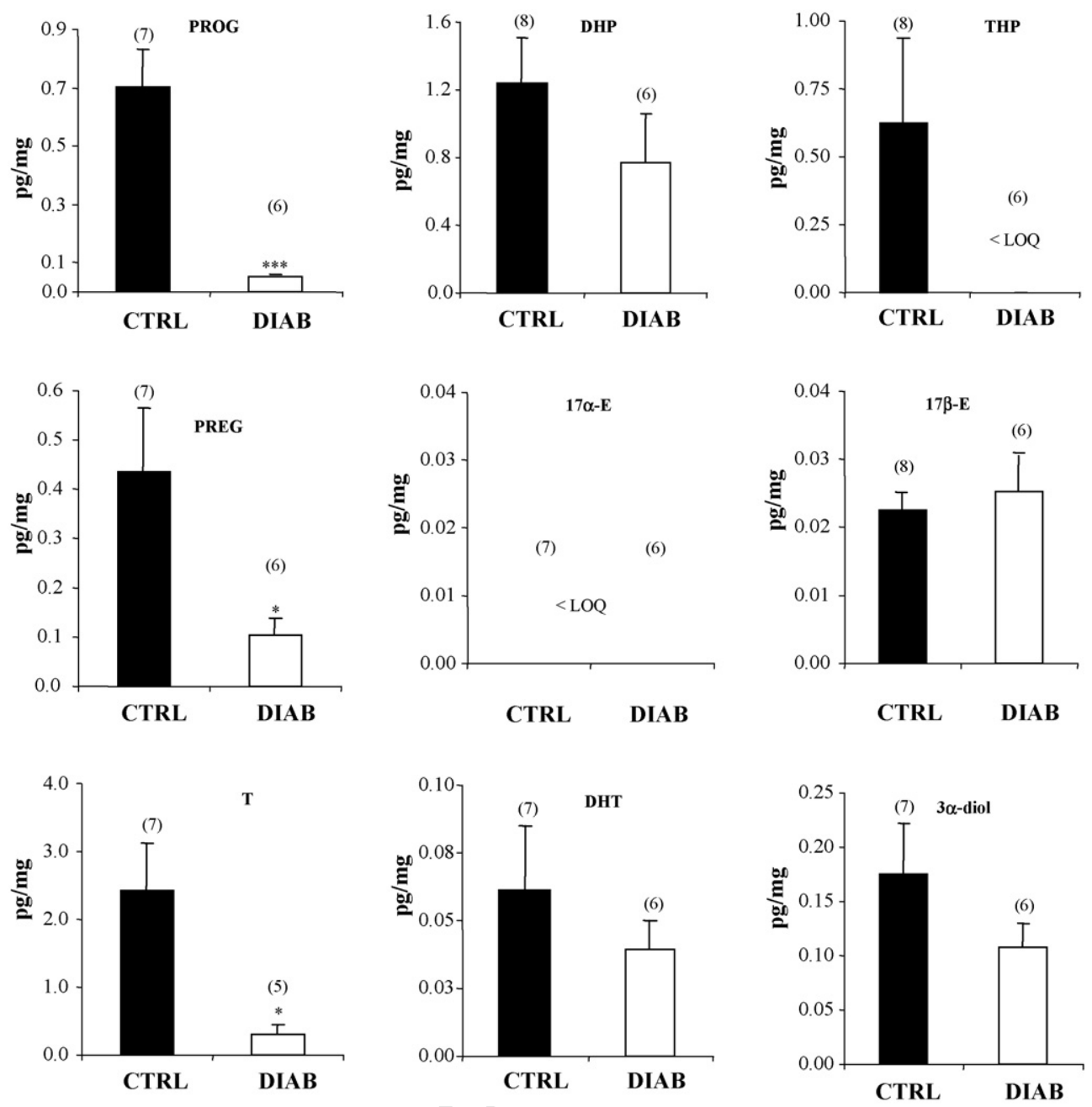

Fig. 2. Effect of diabetes on the levels of neuroactive steroids in the cerebellum. Data are expressed as pg/mg tissue \pm S.E.M. (number of determinations in each group in parentheses). LOQ: limit of quantification (see Table 2 for details). ${ }^{*} p<0.05,{ }^{* * * *} p<0.005$ vs. control (CTRL).

mass spectrometry (MS) by means of atmospheric pressure ionization (API) (e.g., electrospray, ESI and atmospheric pressure chemical ionization, APCI) and in particular the improvements brought about by tandem MS (MS/MS), has opened new perspectives in terms of mass spectrometric identification and quantification of steroids that are difficult to analyze by gas chromatography-MS. With respect to the ionization mode, APCI is mainly applied to rather less polar compounds than ESI but is less susceptible to ion suppression due to the presence of several interferences as is the case in biological tissues. For quantitative assays employing MS detection, triple quadrupole systems are most commonly used, while the new generation of ion trap, namely the linear trap, exhibits similar performance, as also demonstrated by our results. In addition, when an APCI-linear trap is operated in the MS/MS mode, the identification and quantification of the analytes are based on both precursor and product ions, giving higher selectivity and better sensitivity than for any other MS system. Based on these factors, we set up the analytical method reported here, which permitted to simultaneously measure several neuroactive steroids in cerebral cortex, cerebellum, spinal cord and brachial nerve. Data obtained have indicated that these nervous structures did not show the same pattern of distribution of the neuroactive steroids under consideration. For instance, it is interesting to note that only the brachial nerve showed the presence of all these neuroactive steroids (i.e., PREG, PROG, DHP, THP, T, DHT, $3 \alpha$-diol, $17 \alpha$-E and $17 \beta-E$ ). In the central nervous system, the cerebellum showed detectable levels of all these neuroactive steroids with the exception of $17 \alpha$-E. In contrast, the cerebral cortex seems to be unable to produce or accumulate PROG metabolites (i.e., DHP and THP) as well as $17 \alpha$-E and $17 \beta$-E. A similar pattern is evident in the spinal cord, where DHP, $17 \alpha-\mathrm{E}$ and $17 \beta$-E were under detection limits. Levels of PROG metabolites as well as of $17 \alpha-\mathrm{E}$ and $17 \beta-\mathrm{E}$ in cerebral cortex and spinal cord are in apparent disagreement with observations available in the literature indicating that these two nervous structures seem to express the enzymes producing PROG metabolites (i.e., $5 \alpha-$ reductase and $3 \alpha$-hydroxysteroid dehydrogenase (Melcangi et al., 1987; Stoffel-Wagner, 2003; Patte-Mensah et al., 2004; Agis-Balboa et al., 2006) and converting T into estrogens (i.e., aromatase) (Evrard and Balthazart, 2003; Stoffel-Wagner, 2003; Yague et al., 2006). The discrepancy between the local levels of steroids and the local expression of steroidogenic 

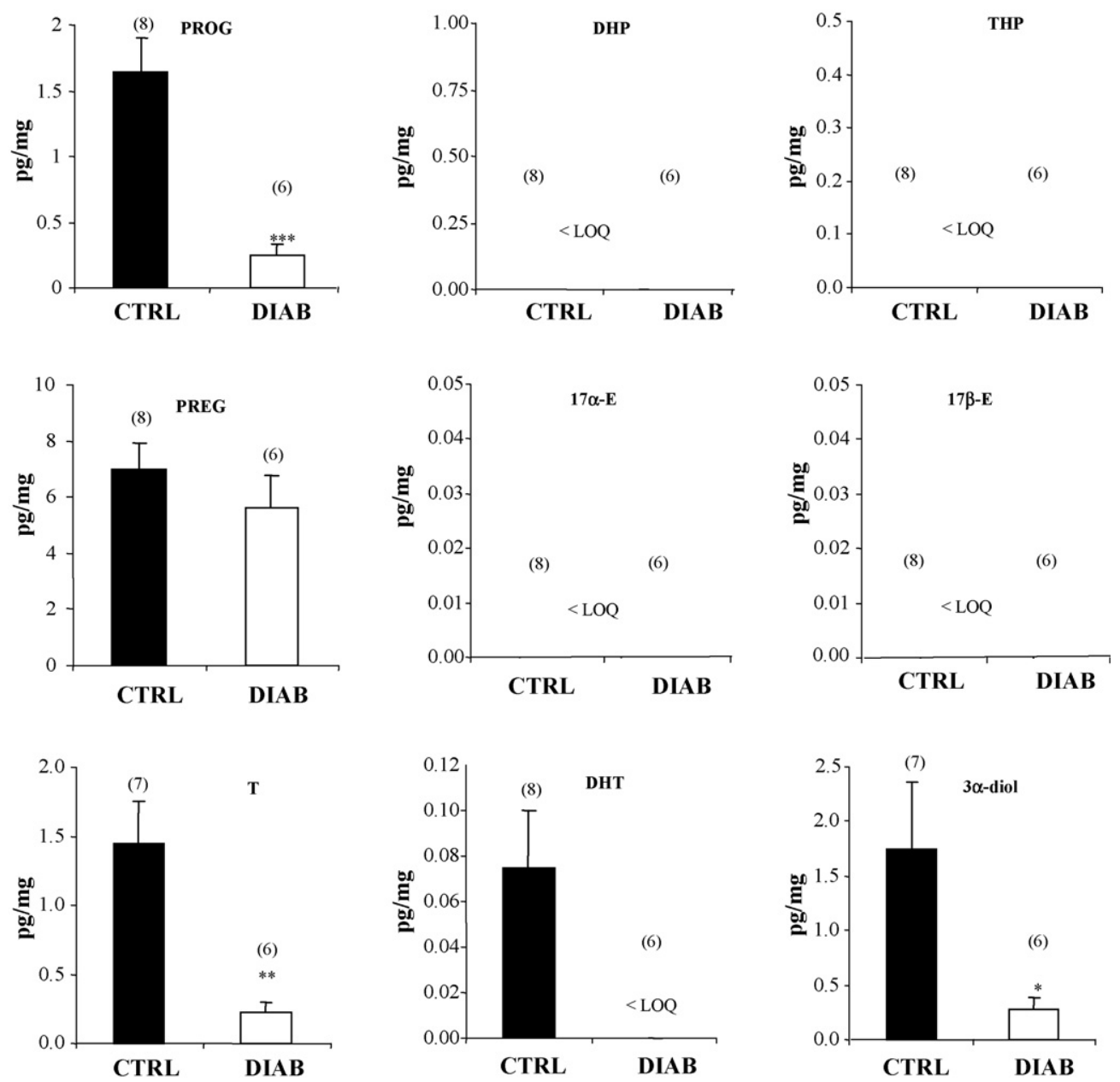

Fig. 3. Effect of diabetes on the levels of neuroactive steroids in the cerebral cortex. Data are expressed as $\mathrm{pg} / \mathrm{mg}$ tissue \pm S.E.M. (number of determinations in each group in parentheses). LOQ: limit of quantification (see Table 2 for details). ${ }^{*} p<0.05,{ }^{* *} p<0.02,{ }^{* * * *} p<0.005$ vs. control (CTRL).

enzymes suggests that PROG metabolites and estrogens are rapidly cleared in the cerebral cortex and the spinal cord. In addition, levels of expression of steroidogenic enzymes may not necessarily reflect their levels of activity.

The results reported here also indicate that diabetes, induced by injection with STZ, strongly affects the levels of neuroactive steroids. Generally a decrease both in the CNS and PNS was observed. In some cases the effects of diabetes on steroid levels in neural tissue did not completely reflect the changes previously reported in plasma. For instance, the levels of PREG in the brachial nerve and cerebellum of diabetic rats reported here show a significant decrease, but remain unchanged in plasma (Leonelli et al., 2007). These findings suggest that diabetes differentially alters steroid synthesis in endocrine glands and nervous structures. Indeed, formation of PREG and other steroids in the peripheral and central nervous systems is not surprising. It has been clearly established that glial cells of the peripheral and central nervous systems express molecules, such as translocator Protein-18 kDa (TSPO, formerly known as peripheral benzodiazepine receptor) and steroidogenic acute regulatory protein, able to participate in the transport of cholesterol to the inner mitochondrial membrane where cytochrome P450scc (i.e., the enzyme forming PREG) is located (Garcia-Segura and Melcangi, 2006). An altered neurosteroidogenesis has been also observed in different forms of neural injury and different neuropathological conditions. For instance, TSPO basal expression is upregulated in gliomas, in neurodegenerative disorders, and in various forms of brain injury and inflammation (Papadopoulos et al., 2006). A very similar effect occurs in the PNS, where the expression of TSPO is increased in Schwann cells after nerve lesion and returns to normal levels when regeneration is completed (Lacor et al., 1999). This induction has been interpreted as reflecting an endogenous increase in steroidogenesis as a neuroprotective response to the damage. In agreement with that, an increased biosynthesis of PROG has been detected in the spinal cord of diabetic animals using HPLC combined with a continuous flow scintillation detection method (HPLC-Flo/one method) utilizing exogenous substrate (tritiated PREG) to evaluate PROG formation (Saredi et al., 2005). It is clear that the endogenous mechanism triggered by diabetes is certainly not enough to protect the nervous system efficiently. Furthermore, although PROG biosynthesis is increased, we demonstrate here that PROG levels are decreased in spinal cord of diabetic animals, suggesting an increased PROG metabolism. In this regard it is also important to note that while the levels of PROG are 

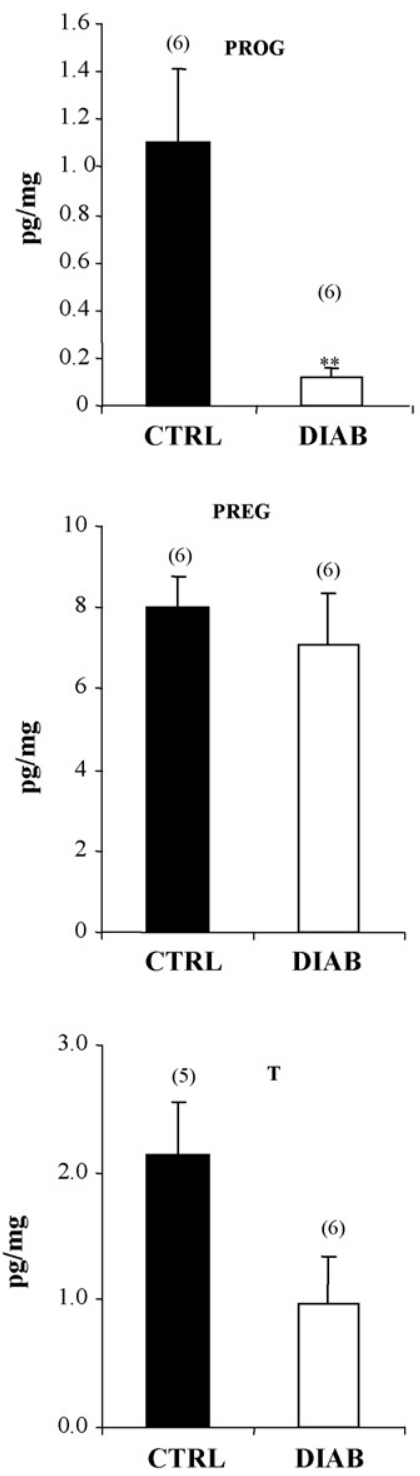
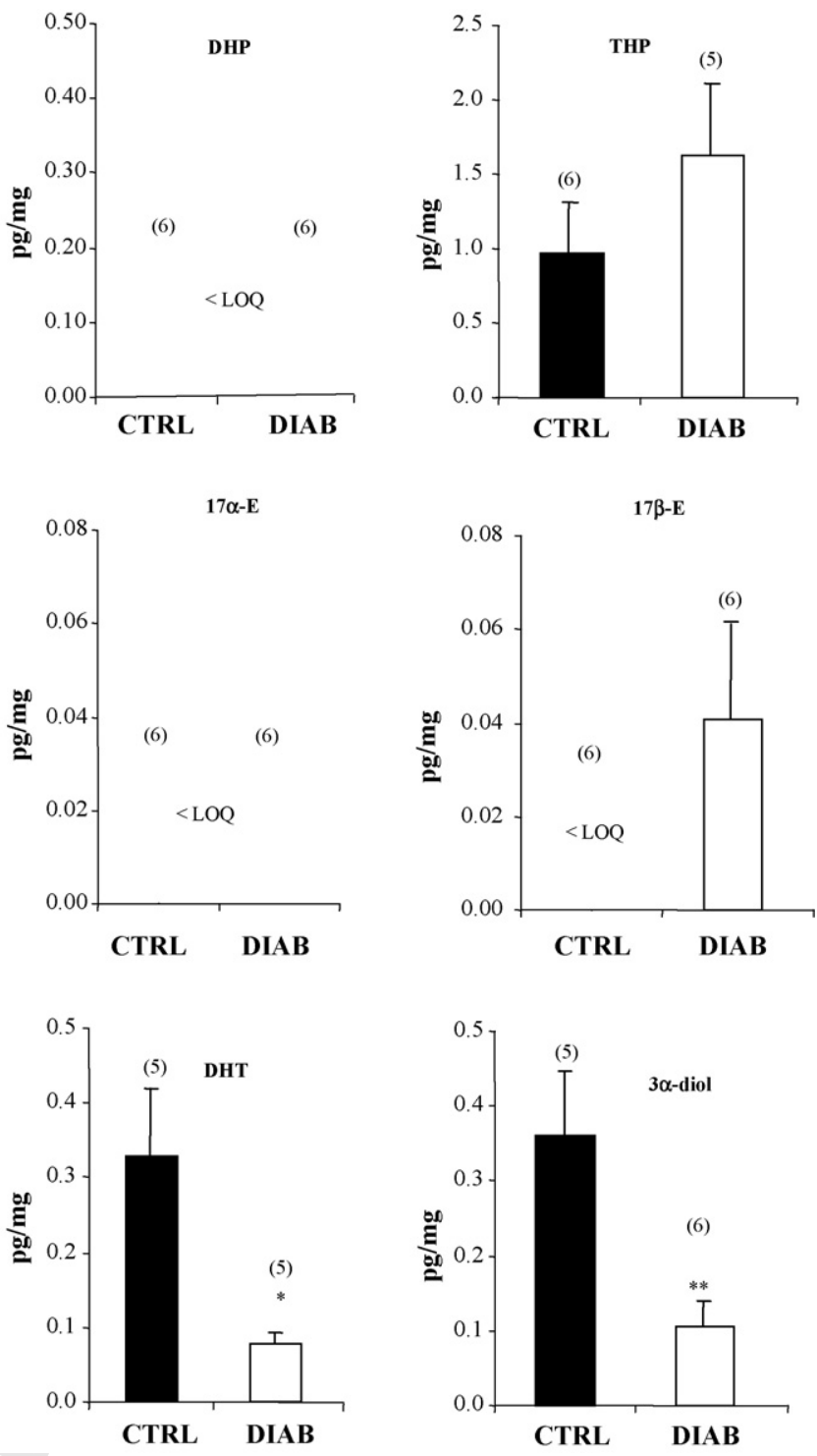

Fig. 4. Effect of diabetes on the levels of neuroactive steroids in the spinal cord. Data are expressed as $\mathrm{pg} / \mathrm{mg}$ tissue \pm S.E.M. (number of determinations in each group in parentheses). LOQ: limit of quantification (see Table 2 for details). ${ }^{*} p<0.05,{ }^{* *} p<0.02$ vs. control (CTRL).

decreased, the levels of its metabolite THP are unaffected by diabetes, further suggesting an enhanced PROG metabolism in the spinal cord of diabetic animals.

The decrease of the levels of several neuroactive steroids associated with diabetes is also interesting in relation with the protective effect exerted by some of these molecules.

Indeed, recent observations have shown that neuroactive steroids might provide a new therapeutic tool for damage induced by diabetes both in PNS and CNS. For instance, dehydroepiandrosterone prevents vascular and neuronal dysfunction in the sciatic nerve of STZ-treated rats (Yorek et al., 2002). In the same experimental model, we recently observed that PROG and its derivatives, DHP and THP, reversed the impairment of nerve conduction velocity and thermal threshold, restored intra-epidermal nerve fiber density, improved $\mathrm{Na}^{+}, \mathrm{K}^{+}$ATPase activity, and counteracted the decrease of gene expression of myelin proteins, such as glycoprotein zero and peripheral myelin protein 22 (Leonelli et al., 2007). We also observed that PROG or DHP administration results in a significant reduction in the number of fibers with myelin abnormalities in the sciatic nerve of STZ-treated rats (Veiga et al., 2006).

Neuroactive steroids are also protective against detrimental effects of diabetes mellitus on the CNS. For instance, estrogens can increase the regional brain glucose utilization in diabetic $(\mathrm{db} / \mathrm{db})$ mice (Garris, 1999). Moreover, as demonstrated in STZ-rats, treatment with $17 \beta$-E may have a beneficial effect in dementia disorders associated with diabetes (Lannert et al., 1998), and, as demonstrated in Bio Breeding (BB) diabetic rats, decreases the infarct size after temporary focal ischemia (Toung et al., 2000). Furthermore, it has been recently demonstrated that $17 \beta-E$ stimulates brain neurogenesis and exerts protective effects at hippocampus level in STZ mice (Saravia et al., 2006).

In conclusion, the data reported here demonstrate that the LC-MS/MS method described allows the assessment of neuroactive steroids in structures of CNS and PNS with high 

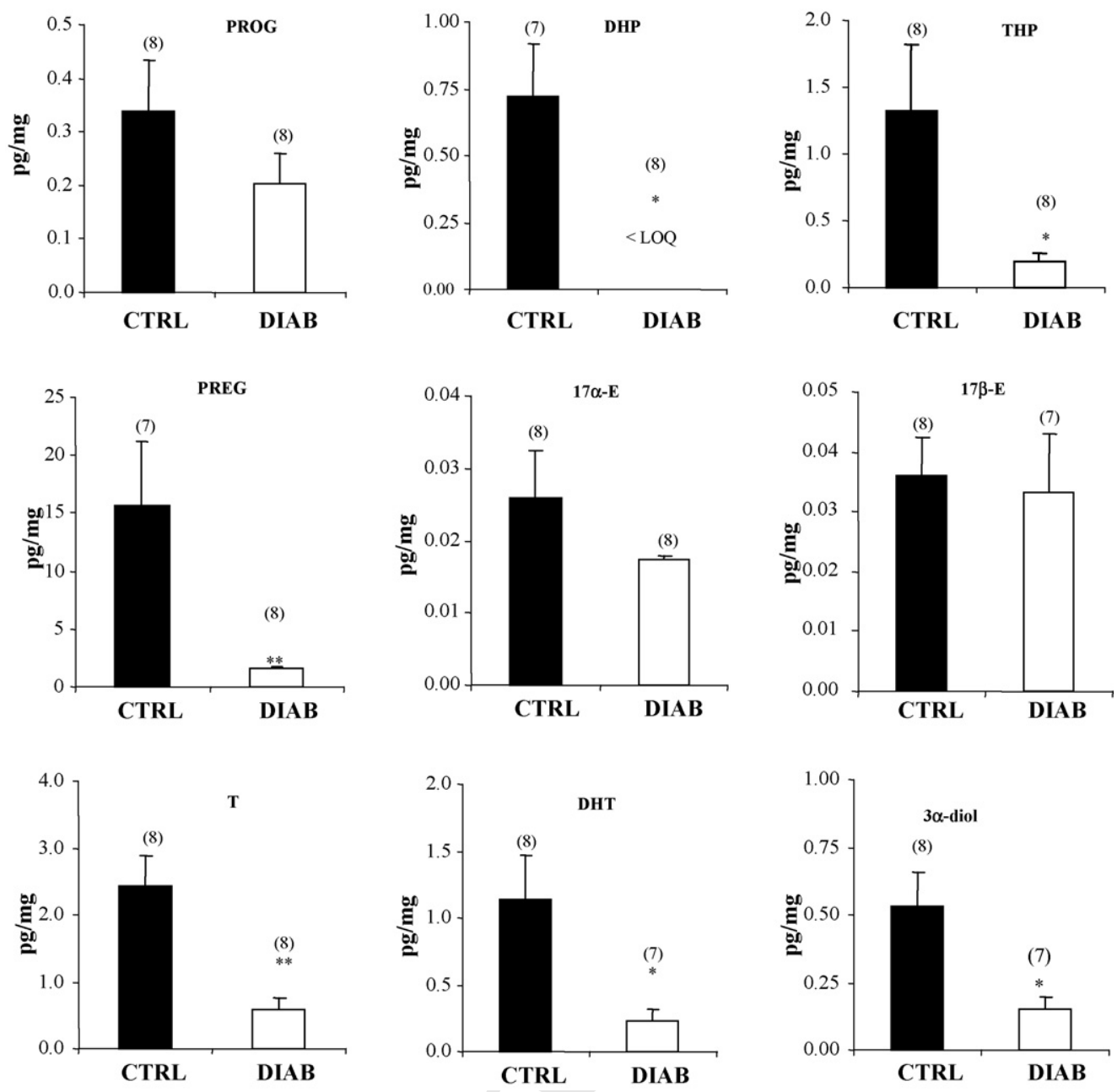

Fig. 5. Effect of diabetes on the levels of neurosteroids in the brachial nerve. Data are expressed as $\mathrm{pg} / \mathrm{mg}$ tissue \pm S.E.M. (number of determinations in each group in parentheses). LOQ: limit of quantification (see Table 2 for details). ${ }^{*} p<0.05,{ }^{* * *} p<0.02$ vs. control (CTRL).

sensitivity and specificity. By means of this methodological procedure, we observed that diabetes strongly affects the levels of several neuroactive steroids in the CNS and PNS. This finding provides a further basis for the proposal of a therapeutic strategy based on neuroactive steroids aimed at counteracting the neurodegenerative effects of diabetes.

\section{Acknowledgements}

The financial supports of PRIN (2005060584_004) and FIRST from University of Milan, Italy to R.C.M. are gratefully acknowledged.

\section{References}

Agis-Balboa, R.C., Pinna, G., Zhubi, A., Maloku, E., Veldic, M., Costa, E., Guidotti, A., 2006. Characterization of brain neurons that express enzymes mediating neurosteroid biosynthesis. Proc. Natl. Acad. Sci. U.S.A. 103, 14602-14607.

Azcoitia, I., Leonelli, E., Magnaghi, V., Veiga, S., Garcia-Segura, L.M., Melcangi, R.C., 2003. Progesterone and its derivatives dihydroprogesterone and tetrahydroprogesterone reduce myelin fiber morphological abnormal- ities and myelin fiber loss in the sciatic nerve of aged rats. Neurobiol. Aging 24, 853-860.

Bianchi, R., Buyukakilli, B., Brines, M., Savino, C., Cavaletti, G., Oggioni, N., Lauria, G., Borgna, M., Lombardi, R., Cimen, B., Comelekoglu, U., Kanik, A., Tataroglu, C., Cerami, A., Ghezzi, P., 2004. Erythropoietin both protects from and reverses experimental diabetic neuropathy. Proc. Natl. Acad. Sci. U.S.A. 101, 823-828.

Biessels, G.-J., Cristino, N.A., Rutten, G.-J., Hamers, F.P.T., Erkelens, D.W., Gispen, W.H., 1999. Neurophysiological changes in the central and peripheral nervous system of streptozotocin-diabetic rats. Brain 122, 757-768.

Ciriza, I., Azcoitia, I., Garcia-Segura, L.M., 2004. Reduced progesterone metabolites protect rat hippocampal neurones from kainic acid excitotoxicity in vivo. J. Neuroendocrinol. 16, 58-63.

El'tseva, T.V., Adamskaya, E.I., Peryshkova, T.A., Babichev, V.N., 1993. Disturbance of neuroendocrine regulation of sexual behavior of male rats with streptozotocin diabetes. Neurosci. Behav. Physiol. 23, 538-544.

Evrard, H.C., Balthazart, J., 2003. Aromatase (estrogen synthase) activity in the dorsal horn of the spinal cord: functional implications. Ann. N.Y. Acad. Sci. 1007, 263-271.

Garcia-Segura, L.M., Melcangi, R.C., 2006. Steroids and glial cell function. Glia 54, 485-498.

Garris, D.R., 1999. Estrogenic stimulation of hypothalamic-limbic system metabolism in ageing diabetic C57BL/KsJmice. Neuroendocrinology 69, $424-429$.

Gispen, W.H., Biessels, G.-J., 2000. Cognition and synaptic plasticity in diabetes mellitus. TINS 23, 542-549. 
Griffin, L.D., Gong, W., Verot, L., Mellon, S.H., 2004. Niemann-pick type C disease involves disrupted neurosteroidogenesis and responds to allopregnanolone. Nat. Med. 10, 704-711.

Jacobson, A.M., Samson, J.A., Weinger, K., Ryan, C.M., 2002. Diabetes, the brain, and the behavior: is there a biological mechanism underlying the association between diabetes and depression? Int. Rev. Neurobiol. 51, 455-479.

Lacor, P., Gandolfo, P., Tonon, M.C., Brault, E., Dalibert, I., Schumacher, M., Benavides, J., Ferzaz, B., 1999. Regulation of the expression of peripheral benzodiazepine receptors and their endogenous ligands during rat sciatic nerve degeneration and regeneration: a role for PBR in neurosteroidogenesis. Brain Res. 815, 70-80.

Lannert, H., Wirtz, P., Schuhmann, V., Galmbacher, R., 1998. Effects of Estradiol (-17beta) on learning, memory and cerebral energy metabolism in male rats after intracerebroventricular administration of streptozotocin. J. Neural. Transm. 105, 1045-1063.

Lapchak, P.A., Araujo, D.M., 2001. Preclinical development of neurosteroids as neuroprotective agents for the treatment of neurodegenerative diseases. Int. Rev. Neurobiol. 46, 379-397.

Leonelli, E., Bianchi, R., Cavaletti, G., Caruso, D., Crippa, D., Garcia-Segura, L.M., Lauria, G., Magnaghi, V., Roglio, I., Melcangi, R.C., 2007. Progesterone and its derivatives are neuroprotective agents in experimental diabetic neuropathy: a multimodal analysis. Neuroscience 144, 1293-1304.

McCullogh, L.D., Hurn, P.D., 2003. Estrogen and ischemic neuroprotection: an integrated view. Trends Endocrinol. Metab. 14, 228-235.

Melcangi, R.C., Celotti, F., Poletti, A., Negri-Cesi, P., Martini, L., 1987. The 5alpha-reductase activity of the subcortical white matter, the cerebral cortex and the hypothalamus of the rat and of the mouse: possible sex differences and effect of castration. Steroids 49, 259-270.

Melcangi, R.C., Magnaghi, V., Galbiati, M., Martini, L., 2001. Formation and effects of neuroactive steroids in the central and peripheral nervous system. Int. Rev. Neurobiol. 46, 145-176.

Melcangi, R.C., Martini, L., Galbiati, M., 2002. Growth factors and steroid hormones: a complex interplay in the hypothalamic control of reproductive functions. Prog. Neurobiol. 67, 421-449.

Melcangi, R.C., Cavarretta, I.T., Ballabio, M., Leonelli, E., Schenone, A., Azcoitia, I., Garcia-Segura, L.M., Magnaghi, V., 2005. Peripheral nerves: a target for the action of neuroactive steroids. Brain Res. Rev. 48, 328-338.

Papadopoulos, V., Baraldi, M., Guilarte, T.R., Knudsen, T.B., Lacapere, J.J., Lindemann, P., Norenberg, M.D., Nutt, D., Weizman, A., Zhang, M.R., Gavish, M., 2006. Translocator protein $(18 \mathrm{kDa})$ : new nomenclature for the peripheral-type benzodiazepine receptor based on its structure and molecular function. Trends Pharmacol. Sci. 27, 402-409.

Patte-Mensah, C., Penning, T.M., Mensah-Nyagan, A.G., 2004. Anatomical and cellular localization of neuroactive 5alpha/3alpha-reduced steroid-synthesizing enzymes in the spinal cord. J. Comp. Neurol. 477, 286-299.

Salonia, A., Lanzi, R., Scavini, M., Pontillo, M., Gatti, E., Petrella, G., Licata, G., Nappi, R.E., Bosi, E., Briganti, A., Rigatti, P., Montorsi, F., 2006. Sexual function and endocrine profile in fertile women with type 1 diabetes. Diabetes Care 29, 312-316.

Saravia, F.E., Beauquis, J., Revsin, Y., Homo-Delarche, F., de Kloet, E.R., De Nicola, A.F., 2006. Hippocampal neuropathology of diabetes mellitus is relieved by estrogen treatment. Cell. Mol. Neurobiol. 26, 943-957.

Saredi, S., Patte-Mensah, C., Melcangi, R.C., Mensah-Nyagan, A.G., 2005. Effect of streptozotocin-induced diabetes on the gene expression and biological activity of 3beta-hydroxysteroid dehydrogenase in the rat spinal cord. Neuroscience 135, 869-877.

Sima, A.A.F., Kamiya, H., Li, Z.G., 2004. Insulin, C-peptide, hyperglicemia, and central nervous system complications in diabetes. Eur. J. Pharmacol. 490, 187-197.

Stoffel-Wagner, B., 2003. Neurosteroid biosynthesis in the human brain and its implications. Ann. N.Y. Acad. Sci. 1007, 64-78.

Sudha, S., Valli, G., Julie, P.M., Arunakaran, J., Govindarajulu, P., Balasubramanian, K., 2000. Influence of streptozotocin-induced diabetes and insulin treatment on the pituitary-testicular axis during sexual maturation in rats. Exp. Clin. Endocrinol. Diabetes 108, 14-20.

Tanaka, M., Nakaya, S., Kumai, T., Watanabe, M., Matsumoto, N., Kobayashi, S., 2001. Impaired testicular function in rats with diet-induced hypercholesterolemia and/or streptozotocin-induced diabetes mellitus. Endocr. Res. 27, 109-117.

Toung, T.K., Hurn, P.D., Traystman, R.J., Sieber, F.E., 2000. Estrogen decreases infarct size after temporary focal ischemia in a genetic model of type 1 diabetes mellitus. Stroke 31, 2701-2706.

Vallée, M., Rivera, J.D., Koob, G.F., Purdy, R.H., Fitzgerald, R.L., 2000. Quantification of neurosteroids in rat plasma and brain following swim stress and allopregnanolone administration using negative chemical ionization gas chromatography/mass spectrometry. Anal. Biochem. 287, 153166.

van Dam, E.W., Dekker, J.M., Lentjes, E.G., Romijn, F.P., Smulders, Y.M., Post, W.J., Romijn, J.A., Krans, H.M., 2003. Steroids in adult men with type 1 diabetes: a tendency to hypogonadism. Diabetes Care 26, 1812-1818.

van Harten, B., De Leeuw, F.R., Weinstein, H.C., 2006. Brain imaging in patients with diabetes. Diabetes Care 29, 2539-2548.

Veiga, S., Leonelli, E., Beelke, M., Garcia-Segura, L.M., Melcangi, R.C., 2006. Neuroactive steroids prevent peripheral myelin alterations induced by diabetes. Neurosci. Lett. 402, 150-153.

Yagihashi, S., 1997. Nerve structural defects in diabetic neuropathy: do animals exhibit similar changes? Neurosci. Res. Commun. 21, 25-32.

Yague, J.G., Munoz, A., de Monasterio-Schrader, P., Defelipe, J., GarciaSegura, L.M., Azcoitia, I., 2006. Aromatase expression in the human temporal cortex. Neuroscience 138, 389-401.

Yorek, M.A., Coppey, L.J., Gellett, J.S., Davidson, E.P., Bing, X., Lund, D.D., Dillon, J.S., 2002. Effect of treatment of diabetic rats with dehydroepiandrosterone on vascular and neural fuction. Am. J. Physiol. Endocrinol. Metab. 283, E1067-E1075. 